\title{
XEDS with SDD-Technology in Scanning Transmission Electron Microscopy
}

\author{
M. Falke*, A. Mogilatenko**, H. Kirmse**, W. Neumann**, C. Brombacher***, M. Albrecht***, \\ A. Bleloch****, G. Tränkle*****, A. Käppel*, R. Terborg*, R. Kroemer*, and M. Rohde* \\ * Bruker-AXS Microanalysis GmbH, Berlin, Germany \\ ** Humboldt University zu Berlin, Germany \\ *** Institute of Physics, University of Technology Chemnitz, Germany \\ **** superSTEM Daresbury, The University of Liverpool, UK \\ ***** Ferdinand Braun Institute, Berlin, Germany
}

Further miniaturisation in many applications and the creation of nanostructures of various shapes causes a growing demand to study element distributions in one to three dimensions on the nm-scale and below. Scanning Transmission electron microscopy (STEM) in combination with high angle annular dark field (HAADF) imaging and spectroscopy methods is a powerful tool to do this.

Electron energy loss spectroscopy (EELS) and energy dispersive x-ray spectroscopy ((X)EDS) are the most common methods used in STEM-mode for elemental mapping. EELS has the advantage of providing information on the bonding environment additionally to element identification and quantification. However, mostly not all elements in question have usable EELS edges in an energy range suitable for one EELS experiment or, the edges of interest do overlap. Thus, complementary, and if multiple elements are to be identified and quantified quickly, EDS is the method of choice.

EDS in TEM, up to now, required liquid nitrogen for cooling the Si(Li) detector. Recently, Peltier cooled silicon drift detectors (SDD) became available. Bruker AXS has successfully adapted the SDD technology for the use in TEM and now a liquid nitrogen free EDS detector is available for TEM and STEM. It has an active area of $30 \mathrm{~mm}^{2}$ and can be arranged to maximise the solid angle and thus the signal. XFlash SDDs have a good light element performance, high energy resolution and show very little dead time, extreme count rate capabilities, and are - unlike most $\mathrm{Si}(\mathrm{Li})$ immune to overload conditions.

The successful performance of an SDD-EDS detector covering a solid angle of 0.12 sr on a conventional TEM/STEM will be demonstrated on various examples of nano-structures. For comparison Fig.1. shows the performance of an SDD covering a solid angle of 0.01 sr only on an SEM. Fig. 2. shows a TEM study of CdZnTe - nanorods with a solid angle of $0.12 \mathrm{sr}\left(30 \mathrm{~mm}^{2}\right.$ SDD). Furthermore, the elemental profile an InGaAs/AlGaP quantum well structure was determined with nm-resolution. The measurement time needed, to achieve useful results, was short (ca 3 minutes for a $40 \mathrm{~nm}$ by $40 \mathrm{~nm}$ region taken with $600 \times 600$ pixels). The element distribution, extracted during the measurement from the raw data, resembles the nominal deposition sequence and correlates with the high angle annular dark field (HAADF) signal. The latter increases monotonically with the atomic number and represents the mass thickness of the sample. Fig. 3. shows, that the data statistics in the already well interpretable fast mapping could be improved considerably using longer measurement times and post drift correction.

As another example, a hedgehog-like magnetic nano-structure on $\mathrm{SiO}_{2}$ spheres was studied using EELS on a Cs-corrected dedicated STEM. EELS showed the distribution of $\mathrm{O}, \mathrm{Si}, \mathrm{Cr}, \mathrm{Co}$ and the 
Ru-seed material. Complementary, with SDD-EDS on an uncorrected conventional TEM/STEM, the Pt distribution could be studied to confirm the Z-contrast data, the composition of the active magnetic layer and to support micromagnetic simulations of the energy barrier, explaining the switching behaviour of the magnetic nano-structures.

The authors thank S. Kret, et al.: Polish Academy of Science for the ZnCdTe nanorod samples.
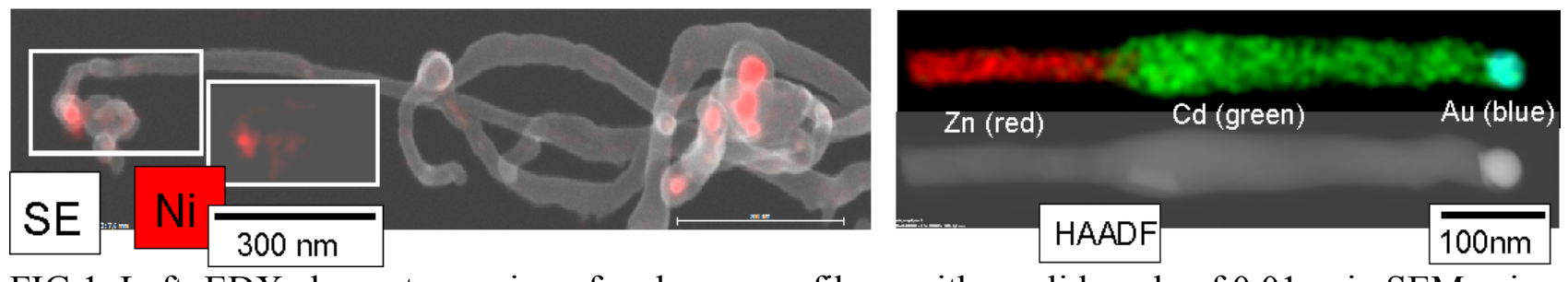

FIG.1. Left: EDX element mapping of carbon nano-fibres with a solid angle of $0.01 \mathrm{sr}$ in SEM using a TEM sample holder and SDD, the inset shows the Ni-signal from marked area. FIG.2.Right:

CdZnTe nano-rod, Cd,Zn,Au-map and HAADF (conventional STEM using SDD-EDS with 0.12 sr).
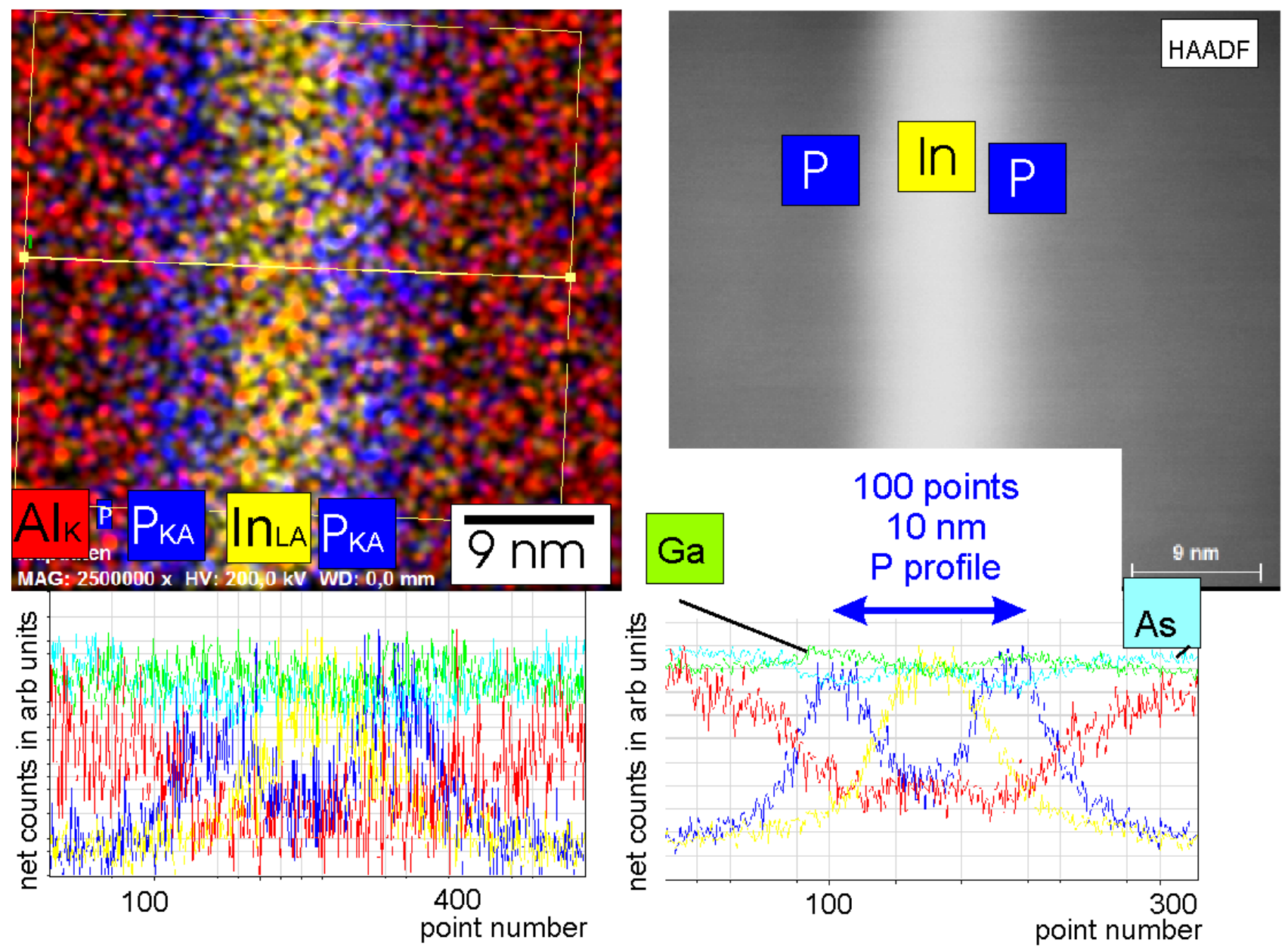

FIG.3. Element map, HAADF and raw element net counts for a quantum well structure using 3 min (left) and $50 \mathrm{~min}$ (right pofile) for the map (conventional STEM, SDD-EDS with $0.12 \mathrm{sr}$, raw data). 